\title{
Assessing Frequency and Clinical Outcomes of BRCA Mutated Ovarian Cancer in Saudi Women
}

Naela Agha ( $\nabla$ dr.nmamdouh@yahoo.com )

Belfast City Hospital

\section{Bader Alshamsan}

King Faisal Specialist Hospital and Research Center

Sharifa Al-Farsi

King Faisal Specialist Hospital and Research Center

Heba Aly Ateya

King Faisal Specialist Hospital and Research Center

Fahed A Almugbel

King Faisal Specialist Hospital and Research Center Hazem Abdullah Alotaibi

King Faisal Specialist Hospital and Research Center

\section{Ayman Omar}

King Faisal Specialist Hospital and Research Center Amgad Shahin Mohamed

King Faisal Specialist Hospital and Research Center

Hanan Alharthy

King Faisal Specialist Hospital and Research Center

\section{Tusneem Elhassan}

King Faisal Specialist Hospital and Research Center

\section{Hany Salem}

King Faisal Specialist Hospital and Research Center

Hamed Alhussainia

King Faisal Specialist Hospital and Research Center

\section{Research}

Keywords: Ovarian Cancer, BRCA1, BRCA2, Germline, BRCA mutation, Founder mutation

Posted Date: January 5th, 2021

DOI: https://doi.org/10.21203/rs.3.rs-126544/v2 
License: (c) (i) This work is licensed under a Creative Commons Attribution 4.0 International License. Read Full License

Version of Record: A version of this preprint was published at BMC Cancer on January 3rd, 2022. See the published version at https://doi.org/10.1186/s12885-021-09123-6. 


\section{Abstract}

Purpose: BRCA gene mutations (BRCAm) had an impact on patients' characteristics and clinical outcomes of ovarian cancer (OC). The frequency and patterns of BRCAm vary among countries and ethnicities. There are limited data from Saudi Arabia (SA); thus, this study aims to determine the frequency, pattern, and impact on patient characteristics and outcomes of BRCAm OC compared to wildtype BRCA (BRCAw) in Saudi women.

Methods: This retrospective study evaluated women diagnosed with non-mucinous OC, fallopian tube, or peritoneal carcinoma who had BRCA status tested in an accredited lab between January 2016 and December 2017. The associations between various parameters and BRCAm were estimated using logistic regression. Statistical analysis performed with SPSS (Version 27).

Result: Sixty-one women with a median age of 52 at diagnosis were analyzed. Germline BRCA mutations were found in $41 \%$ of cases $(25 / 61)$. The most common deleterious germline BRCA1 mutation was c. 1140 dupG $(39 \%)$. Most women $(72 \%)$ had no family history of cancers and $82 \%$ had advanced stage. Regardless of BRCA mutations, an optimal overall response rate (ORR) to first-line treatment has been achieved although most cases relapsed (84\%) and the majority were platinum-sensitive relapse (85\%). Higher ORR to subsequent lines and better survival were obtained in women with BRCA-mutation.

Conclusion: The prevalence of BRCAm of OC was higher in Saudi women compared to regional and most of the international figures. The better clinical outcomes of BRCAm women agreed with the reported evidence. Further studies on BRCA mutations of $\mathrm{OC}$ and genetic counseling are highly recommended.

\section{Trial registration number and The addresses of the institution at which the work was performed:}

Trial approved by the Institutional Review Board of King Faisal Specialist Hospital and Research Center (RAC \# 2171137) and conducted at King Faisal Specialist Hospital and Research Center, PO Box 3354, Riyadh 11211, Saudi Arabia.

\section{Background}

In high-income countries, ovarian cancer $(\mathrm{OC})$ is the second most commonly diagnosed gynecological malignancy and the most common cause of death from it [1]. The age-standardized rate (ASR) incidence of OC worldwide is 3.9 per 100,000, and in Arab regions, it ranges between 0.9 and 8 per 100,000 [1, 2]. In Saudi Arabia (SA), OC ranks as the seventh most common cancer among females, with ASR of 3.7 cases per 100,000 and as the fifth leading cause of death, with an ASR of 2.5 deaths per $100,000[3,4]$. The inherited mutations in $\mathrm{OC}$ were found to be the cause for $18 \%$ of the $\mathrm{OC}$ cases, with the majority being caused by breast cancer associated gene mutations (BRCAm). Furthermore, BRCAm increases the lifetime risk of developing OC from $1.3 \%$ in the general population to $44 \%$ in BRCA1 and $17 \%$ in BRCA2 mutation carriers [5]. A review of 173 women with breast cancer and OC from SA showed that the prevalence of germline BRCAm was $30.7 \%$ in OC and $10.2 \%$ in breast cancer [6]. In a recent systemic 
review from 22 Arab countries, six mutations were shown to be unique to the Saudi population: Four were located in BRCA1 (c.1140dupG, c.5530delC, c.5054C>T, and c.711_712insTGAA), and two were located in BRCA2 (c.2667delT and c.5760_5770del11) [2]. BRCA1 and BRCA2 are tumor suppressor genes that control cell growth and maintain genomic stability [7]. They are responsible for the repair of double-strand DNA breaks (DSBs) through the homologous recombination pathway, and a deficiency in BRCA function leads to genomic instability due to the inability to repair DNA damage through homologous recombination defect (HRD), thereby leading to tumorigenesis [8, 9]. Simultaneously, the mutation weakens tumor cells that can be targeted therapeutically [10], which explains the association between BRCA gene mutations and a better response to DNA-alkylating agents such as platinum in $O C[8,11]$. Poly ADP-ribose polymerase enzyme (PARP) is essential for the repair of single-strand breaks of DNA. PARP inhibitors induce the synthetic lethality of cells with HRD, which occurs when there is a simultaneous mutation in two genes leading to cell death; however, no cell death occurs when the mutation is found in only one gene [10]. Many clinical trials have tested the efficacy of PARP inhibitors in the treatment of advanced OC. Early evidence showed improvement in objective response rate (ORR) and progression-free survival (PFS) in heavily treated patients, predominantly in those with either germline or somatic BRCA mutations as HRD occurs in germline BRCA mutations, somatic BRCA mutations, and BRCA promotor hypermethylation cases [12]. Moreover, this benefit has recently been proven to involve all women with advanced high-grade serous OC after response to platinum-based chemotherapy (PBC) regardless of BRCA status [13]. The standard therapy for advanced epithelial OC is PBC following primary debulking surgery (PDS) [14]. Interval debulking surgery (IDS) is a feasible option after neoadjuvant chemotherapy and demonstrated similar survival outcomes as PDS [15]. However, more than $80 \%$ of patients experience disease recurrence after completing their treatment, with an unsatisfactory outcome to the second line of management [16].

The primary aim of this study was to measure the frequency and patterns of germline BRCA mutations among $O C$ patients, to compare the clinicopathological characteristics, and to assess the clinical outcomes of BRCA mutant vs BRCA wild-type patients.

\section{Methods}

A retrospective study was conducted on all patients diagnosed with primary ovarian, fallopian tube, or peritoneal carcinoma who were tested for BRCA mutations and followed up at the King Abdullah Oncology Centre at the King Faisal Specialist Hospital and Research Center (KFSH\&RC) between January 2016 and December 2017. Women with pathological confirmation of serous carcinoma, clear cell carcinoma, and endometrial carcinoma were eligible, whereas those with borderline cancer and mucinous carcinoma were excluded. Germline BRCA mutations were obtained from Myriad Genetic Laboratories Inc., which was certified by the College of American Pathologists and Clinical Laboratory Improvement Amendments as per the National Comprehensive Cancer Network (NCCN) guidelines [17]. This project was conducted in accordance with the ethical principles in Helsinki's Declaration (2000) and was approved by the Institutional Review Board of KFSH\&RC (RAC \# 2171137). The clinical information collected from the medical records included age, personal and family history of cancer, tumor histology, 
grade, Federation of Gynecology and Obstetrics (FIGO) stage, CA125 level, and BRCA status. In addition to lines of chemotherapy, best response, time to progression, platinum sensitivity, and status at last follow-up were evaluated. The tumors were staged according to the $20178^{\text {th }}$ Edition of the American Joint Committee on Cancer and the FIGO classification system. The patients were followed up based on KFSH\&RC guidelines (every two to three months for the first two years and then every six months). At each visit, clinical assessment and serum CA125 test were performed. The abdominal ultrasound scans were performed every six months, and computed tomography of the chest and abdomen was done every year for five years unless relapse was suspected. Platinum-sensitive relapse was defined as tumor relapse that occurred more than six months after completion of the last cycle of PBC. Tumor response was assessed based on the Response Evaluation Criteria in Solid Tumors Version 1.1 (RECIST 1.1). The clinical outcomes investigated were over all response rate (ORR) to first and subsequent lines, diseasefree survival (DFS), and overall survival (OS). The ORR has been defined as the sum of partial and complete responses divided by the total number of patients. DFS was defined as the interval between histologic diagnosis and first progression, death as a result of disease, or last follow-up. OS was defined as the interval between histologic diagnosis and the date of death as a result of disease or last follow-up.

\section{Statistical analysis}

Categorical values were described as frequencies compared with Chi-square test or Fisher's exact test, and continuous values were described as the median with interquartile range (IQR) and compared using the Mann-Whitney $\mathrm{U}$ test. Associations between various parameters and the BRCA mutations were estimated by logistic regression. The Kaplan-Meier estimator was used to determine DFS, and OS and survival curves were compared using the log-rank test, and a multivariant analysis was conducted using the Cox regression. All variables were tested for the affirmation of the proportionality assumption. Variables that violated the proportionality assumption were entered as time-dependent covariates. BRCA mutations were considered the main effect and were kept in the model at all times. Interactions between BRCA mutations and other significant variables were evaluated, and significant interactions were considered. A p-value of $\leq 0.05$ was considered statistically significant. The SPSS for Mac, v27; IBM Corp, Armonk, NY, USA, was performed for statistical analysis.

\section{Results}

A total of 61 women were eligible for analysis. The median age at diagnosis was 52 years (IQR: 4461.5). BRCA mutations were found in 25 women (41\%), including 23 with BRCA1 mutations and 2 with BRCA2 mutations. The patient and disease characteristics stratified by BRCA status are shown in Table 1. The main presenting symptom was abdominal distension (35 patients, 57.3\%). The logistic regression revealed a significant association between family history of malignancy and BRCA mutations $(p=0.03)$. However, BRCA mutations were not statistically significantly associated with age and stage at diagnosis, patient region, or history of primary cancer. 
There were 15 different pathogenic variants identified, including 13 with BRCA1 mutations and 2 with BRCA2 mutations.he three most common deleterious germline BRCA1 mutations were c.1140dupG (9 patients, 39\%), c.5530del (3 patients, 13\%), and c.5095C>T (2 patients, 8\%). The other pathogenic variants were each observed once. Table 2 presents all pathogenic variants of the mutated genes, age, geographical region of the patients, and personal and family histories of cancer. The univariant analysis revealed no association between c.1140dupG, the most common deleterious mutation, and age, stage at diagnosis, relapse rate, platinum sensitivity, or patient region; however, all the OC cases from the western province (3 patients) and 29\% (4 patients) from the central region carried the c.1140dupG pathogenic variant of the BRCA1 gene mutation. Of the BRCA wild-type patients, two had a positive family history of cancer, including a mother with a brain tumor and a sister with colon cancer; one patient had a personal history of cervical cancer.

All women in this cohort underwent debulking surgery and received chemotherapy during their treatment; 49\% received PDS and then adjuvant chemotherapy, and 51\% started with neoadjuvant chemotherapy and IDS with no statistical difference $(p=0.98)$. All women received PBC as the first-line treatment. A total of $87 \%$ of both groups received a regimen consisting of IV cisplatin and paclitaxel every three weeks. The patients received a median of three chemotherapy lines; specifically, $21 \%, 17 \%$, and $62 \%$ of patients received one, two, and three or more chemotherapy lines, respectively. The platinum sensitivity dropped with subsequent lines; $14 \%, 41 \%$, and $73 \%$ of women received non-platinum-based therapy as the secondline, third-line, and fourth-line treatments, respectively, and 100\% (13 patients) received non-platinumbased therapy as the fifth-line treatment. The ORR to the first line of management was $100 \%$, with a higher complete response (CR) in BRCA mutant women than in wild-type women ( $92 \%$ vs $72.7 \%, p=$ 0.08). The relapse rate was $84 \%$, and the majority (85\%) were platinum-sensitive. Additionally, $84 \%$ of BRCA mutant vs. $80 \%$ of BRCA wild-type patients experienced platinum-sensitive relapse after first-line therapy $(p=0.43)$. In the subsequent lines of treatment, the ORR also was higher in the BRCA mutant group compared with the BRCA wild-type group: second-line (94.4\% vs $64.5 \%, p=0.01)$, third-line (84.7\% vs $30.6 \%, p=0.002)$, and fourth-line ( $40 \%$ vs $22.2 \%, p=0.40)$ (Table 3$)$. Thirteen out of 25 women with BRCA mutation received a PARP-inhibitor, namely Olaparib, and over half of the patients received Olaparib after third relapse. $(54 \%, n=7)$. Olaparib was discontinued due to disease progression in eleven patients and anemia in one patient. Olaparib treatment was still ongoing for one patient. The sample size limited further analysis.

The median follow-up duration was 59 months (IQR: 42-79). The median DFS was longer in the BRCA mutant women 25 (95\% Cl: 21.7-28.2) vs. 17 (95\% Cl: 8.7-25) months, p = 0.02) (Fig. 1). The Cox regression analysis for DFS adjusted by age and comorbidities was statistically significant for BRCA mutant vs. wild-type patients (hazard ratio $(\mathrm{HR})=0.52,95 \% \mathrm{Cl}: 0.23-0.92, \mathrm{p}=0.02$ ). The median DFS of second-line treatment (50 patients) in the BRCA mutant group was 20 months (95\% Cl: 18.2-21.7) vs. 12 months (95\% Cl: 7.8-16.1) in the wild-type group ( $p=0.051)$ (Fig. 2). The median OS was not reached. However, the five-year OS rate for BRCA mutant patients was $90.9 \%$ vs. $66.7 \%$ for wild-type patients ( $p=$ 0.19) (Fig. 3). 


\section{Discussion}

This is the first study to compare the clinical characteristics and clinical outcomes of BRCAm and BRCAw in Saudi women with OC. The research highlights the higher prevalence, the better clinical outcomes of BRCA mutants, and the importance of early access for BRCA testing and treatment. The frequency of BRCA-associated OC was higher in this current cohort than in a previous study ( $40 \%$ vs. $30 \%$ ) [6]. It remains unknown whether the high intermarriage between families in SA could explain why Saudi women have a high prevalence of germline BRCA mutations or whether some other factor is involved. The frequency in this cohort is close to the BRCA gene mutations rate found among Ashkenazi Jewish (41\%) [18] and Italian women $(39.2 \%-43.5 \%)[19,20]$. The most common mutations in this study are c. 1140 dupG, c.5530del, and c.5095C $>$ T, which agrees with recent reports $[2,6]$. However, the most common mutations in the Ashkenazi are 185delAG and 5382insC [18] and those in the Italian women are 3875 del4 and 1479delAG [19]. The higher percentage of BRCA1 mutations compared with BRCA2 mutations ( $92 \%$ vs $8 \%$ ) in this study agrees with previous publications although those studies reported higher percentages of BRCA2 mutations $[19,21]$. This trend is reversed in some Asian populations, with a higher percentage of BRCA2 mutations compared with BRCA1 mutations [22]. Clearly, the pattern and frequency of BRCA mutations vary significantly in relation to race/ethnicity and geographical location [23].

The median age at diagnosis was lower than that in other regions: 52 vs 63 years; however, there was no statistically significant difference between BRCAm and BRCAw ( $p$-value $=0.13)$ [24]. There was a greater association of a family history of malignancy and a personal history of cancer with the BRCA mutant patients than in the wild-type patients ( $p=0.02$ and $p=0.04$, respectively), which has been reported earlier [25]. However, most cases of OC did not have a positive family history, as noted in earlier studies [21]. Therefore, the NCCN guidelines recommend susceptibility gene screening regardless of family history for all epithelial OC cases, including fallopian tube cancer or peritoneal cancer diagnosed at any age [17], which has been followed in many institutions [19]. Agreeing with previous studies, abdominal distension the most common presenting symptom in this cohort, and there was no significant difference between BRCAm and BRCAw in terms of FIGO stage at presentation and CA-125 level $[21,26]$.

The higher ORR to subsequent lines of treatment in the BRCA mutant group agrees with earlier evidence showing that BRCA mutations increase the susceptibility of the cells to be destroyed by chemotherapy [11]. All patients received intravenous doublet PBC in a first-line setting, in agreement with the international standard of care [27, 28]. Intraperitoneal chemotherapy is not approved at KFSHRC as the standard treatment because of its high toxicity profile and worsening quality of life [29].

The higher DFS and OS in BRCA gene mutations have been shown in several previous studies, and this finding corresponds to a better response to chemotherapy owing to the deficiency of mechanisms of DNA repair [30]. This trend was clear in this cohort, which had a prolonged median DFS in the BRCA mutant group ( 25 vs. 17 months) in the first-line setting, which was statistically significant. Additionally, the median DFS after second-line treatment was eight months longer in the BRCA mutant group (Fig. 2), and 
the five-year OS was $90 \%$ vs $66 \%$ (Fig. 3) in the BRCA mutant group compared to BRCA wild group; however, this difference was not statistically significant, However, longer follow-up is necessary.

We acknowledge that the small sample size likely yielded underpowered comparisons. Moreover, these patients were recruited from a tertiary referral center and therefore may not be representative of patients at primary cancer centers, although they were obtained from the largest referral hospital in the region.

\section{Conclusions}

BRCA gene mutations in Saudi women with OC predominantly involve the BRCA1 gene. The founder mutation was c.1140dupG, which was observed in more than one-third of the cases. BRCAm women had a better ORR in subsequent lines of therapy and a longer DFS than the BRCAw-type women.

\section{List Of Abbreviations}

ASR

CR

DFS

DSB

EOC

HRD

IDS

KFSH

NCCN

OC

ORR Objective response rate

OS

PARP

PFS

SA

FIGO

Age-standardized rate

Complete response

Disease-free survival

Double-strand breaks

Ovarian cancer

Overall survival

Saudi Arabia
Epithelial ovarian cancer

Homologous recombination defect

Interval debulking surgery

King Faisal Specialist Hospital

National Comprehensive Cancer Network

Poly ADP-ribose polymerase

Progression-free survival

Federation of Gynecology and Obstetrics 
PBC Platinum-based chemotherapy

PDS Primary debulking surgery

\section{Declarations}

\section{Ethics approval and consent to participate}

This project was conducted in accordance with the ethical principles contained in the Declaration of Helsinki (2000). The Research Advisory Council (RAC) at King Faisal Specialist Hospital \& Research Centre, Riyadh, approved the project (RAC \# 2171137), and waiver informed consent includes access and use of patient files while maintaining the confidentiality of the data. The identity of the patients remained anonymous since no identifying data or protected health information was recorded.

\section{Consent for publication}

Not applicable

\section{Availability of data and materials}

The datasets used and analyzed during the current study are available from the corresponding author on reasonable request.

\section{Competing interests}

The authors declare that they have no competing interests.

\section{Funding}

This research did not receive any specific grant from funding agencies in the public, commercial, or notfor-profit sectors.

\section{Author Contributions}

$\mathrm{HH}, \mathrm{HS}, \mathrm{NA}$, and SA shared the idea and wrote the proposal. NA, SA, BS, HA, AS, AO, TM, HO, and HA collected the data. BA and NA formulated the idea, performed the literature review, and wrote the first draft. TE and BA analyzed the data. BA and NA created the tables and figures and edited the manuscript. $\mathrm{HH}$ master supervised the patient management and performed the literature review. All authors read and approved the final version of the manuscript.

\section{References}

[1] Ferlay J, Colombet M, Soerjomataram I, Mathers C, Parkin DM, Piñeros M, et al. Estimating the global cancer incidence and mortality in 2018: GLOBOCAN sources and methods. Int J Cancer. 2019;144:1941- 
53.

[2] Younes N, Zayed H. Genetic epidemiology of ovarian cancer in the 22 Arab countries: a systematic review. Gene. 2019;684:154-64.

[3] Cancer Today [Internet]. [cited 2020 Jul 12]. Available from: https://gco.iarc.fr/today/online-analysismap

[4] A. Wafa, Ahmed. Kingdom m of Saud di Arabia a Saudi Health Council National Health Information Center Saudi Cancer Registry Cancer Incidence Report Saudi Arabia. 2016. Available from: https://nhic.gov.sa/en/eServices/Pages/TumorRegistration.aspx. Date of accession 05-08-2020.

[5] Kuchenbaecker KB, Hopper JL, Barnes DR, Phillips KA, Mooij TM, Roos-Blom MJ, et al. Risks of breast, ovarian, and contralateral breast cancer for BRCA1 and BRCA2 mutation carriers. JAMA. 2017;317:240216.

[6] Alhuqail AJ, Alzahrani A, Almubarak H, Al-Qadheeb S, Alghofaili L, Almoghrabi N, et al. High prevalence of deleterious BRCA1 and BRCA2 germline mutations in arab breast and ovarian cancer patients. Breast Cancer Res Treat. 2018;168:695-702.

[7] Yoshida K, Miki Y. Role of BRCA1 and BRCA2 as regulators of DNA repair, transcription, and cell cycle in response to DNA damage. Cancer Sci. 2004;95:866-71.

[8] Deng CX, Scott F. Role of the tumor suppressor gene Brca1 in genetic stability and mammary gland tumor formation. Oncogene. 2000;19:1059-64.

[9] Tutt A, Bertwistle D, Valentine J, Gabriel A, Swift S, Ross G, et al. Mutation in Brca2 stimulates errorprone homology-directed repair of DNA double-strand breaks occurring between repeated sequences. EMBO J. 2001;20:4704-16.

[10] Ashworth A. A synthetic lethal therapeutic approach: poly(ADP) ribose polymerase inhibitors for the treatment of cancers deficient in DNA double-strand break repair. J Clin Oncol. 2008;26:3785-90.

[11] Wan B, Dai L, Wang L, Zhang Y, Huang H, Qian G, et al. Knockdown of BRCA2 enhances cisplatin and cisplatin-induced autophagy in ovarian cancer cells. Endocr Relat Cancer. 2018;25:69-82.

[12] Moschetta M, George A, Kaye SB, Banerjee S. BRCA somatic mutations and epigenetic BRCA modifications in serous ovarian cancer. Ann Oncol. 2016;27:1449-55.

[13] Mirza MR, Monk BJ, Herrstedt J, Oza AM, Mahner S, Redondo A, et al. Niraparib maintenance therapy in platinum-sensitive, recurrent ovarian cancer. Obstet Gynecol Surv. 2017;72:91-2.

[14] Covens A, Carey M, Bryson P, Verma S, Fung MF, Johnston M. Systematic review of first-line chemotherapy for newly diagnosed postoperative patients with stage II, III, or IV epithelial ovarian cancer. 
Gynecol Oncol. 2002;85:71-80.

[15] Vergote I, Amant F, Kristensen G, Ehlen T, Reed NS, Casado A. Primary surgery or neoadjuvant chemotherapy followed by interval debulking surgery in advanced ovarian cancer. Eur $\mathrm{J}$ Cancer. 2011;47:S88-92.

[16] Gadducci A, Sartori E, Maggino T, Zola P, Landoni F, Fanucchi A, et al. Analysis of failures in patients with stage I ovarian cancer: an Italian multicenter study. Int J Gynecol Cancer. 1997;7:445-50.

[17] Pilarski R, Berry MP, Jude S, Buys SS, Dickson P, Domchek SM, et al. NCCN Guidelines Version 1.2020 Genetic/Familial High-Risk Assessment: Breast, Ovarian, and Pancreatic. https://www.nccn.org/professionals/physician_gls/default.aspx date of accession 05-08-2020.

[18] Moslehi R, Chu W, Karlan B, Fishman D, Risch H, Fields A, et al. BRCA1 and BRCA2 mutation analysis of 208 Ashkenazi Jewish women with ovarian cancer. Am J Hum Genet. 2000;66:1259-72.

[19] Zhang S, Royer R, Li S, Mclaughlin JR, Rosen B, Risch HA, et al. Gynecologic oncology frequencies of BRCA1 and BRCA2 mutations among 1,342 unselected patients with invasive ovarian cancer. Gynecol Oncol. 2011;121:353-7.

[20] Petrillo M, Marchetti C, Leo R De, Musella A, Capoluongo E. BRCA mutational status, initial disease presentation, and clinical outcome in high-grade serous advanced ovarian cancer: a multicenter study. Am J Obstet Gynecol. 2017;217:334.e1-334.e9.

[21] Alsop K, Fereday S, Meldrum C, DeFazio A, Emmanuel C, George J, et al. BRCA mutation frequency and patterns of treatment response in BRCA mutation-positive women with ovarian cancer: a report from the Australian ovarian cancer study group. J Clin Oncol. 2012;30:2654-63.

[22] Kim H, Choi DH. Distribution of BRCA1 and BRCA2 mutations in Asian patients with breast cancer. J Breast Cancer. 2013;16:357-65.

[23] Holschneider, Christine H., and Jonathan S. Berek. Ovarian cancer: epidemiology, biology, and prognostic factors. Seminars in surgical oncology. Vol. 19. No. 1. New York: John Wiley \& Sons, Inc., 2000. p.3-4

[24] Kurian AW. BRCA1 and BRCA2 mutations across race and ethnicity: distribution and clinical implications. Curr Opin Obstet Gynecol. 2010;22:72-8.

[25] Unni SK, Schauerhamer MB, Deka R, Tyczynski JE, Fernandes AW, Stevens V, et al. BRCA testing, treatment patterns and survival in platinum-sensitive recurrent ovarian cancer - An observational cohort study. J Ovarian Res. 2016;9:1-9.

[26] Goff BA, Mandel LS, Melancon CH, Muntz HG. Frequency of symptoms of ovarian cancer in women presenting to primary care clinics. J Am Med Assoc. 2004;291:2705-12. 
[27] Aabo K, Adams M, Adnitt P, Alberts DS, Athanazziou A, Barley V, et al. Chemotherapy in advanced ovarian cancer: four systematic meta-analyses of individual patient data from 37 randomized trials. $\mathrm{Br} \mathrm{J}$ Cancer. 1998;78:1479-87.

[28] Trimbos JB, Vergote I, Bolis G, Vermorken JB, Mangioni C, Madronal C, et al. Impact of adjuvant chemotherapy and surgical staging in early-stage ovarian carcinoma: European organisation for research and treatment of cancer-adjuvant chemotherapy in ovarian neoplasm-trial. J Natl Cancer Inst. 2003;95:113-25.

[29] Jones HW. Intraperitoneal cisplatin and paclitaxel in ovarian cancer: Commentary. Obstet Gynecol Surv. 2006;61:240-2.

[30] Vencken PMLH, Kriege M, Hoogwerf D, Beugelink S, van der Burg MEL, Hooning MJ, et al. Chemosensitivity and outcome of BRCA1- and BRCA2-associated ovarian cancer patients after first-line chemotherapy compared with sporadic ovarian cancer patients. Ann Oncol. 2011;22:1346-52.

\section{Tables}

Table 1. Patient, disease, and treatment characteristics stratified by BRCA status $(n=61)$ 


\begin{tabular}{|c|c|c|c|c|}
\hline & & $\begin{array}{l}\text { BRCA mutant } \\
n=25(41 \%)\end{array}$ & $\begin{array}{l}\text { BRCA wild type } \\
n=36(59 \%)\end{array}$ & \\
\hline \multirow{2}{*}{\multicolumn{2}{|c|}{ Characteristics }} & & & $P$-VALUE* \\
\hline & & N (Frequency) & N (Frequency) & \\
\hline \multicolumn{5}{|l|}{ Age at diagnosis } \\
\hline Median (IQR) & & $50(43-56)$ & $55(46-66)$ & 0.13 \\
\hline Age $\leq 50$ & & $14(56)$ & $15(41.7)$ & 0.27 \\
\hline Positive personal history of cancer & & $5(20)$ & $1(2.9)$ & 0.04 \\
\hline Positive family history of cancer & & $7(28)$ & $2(5.6)$ & 0.02 \\
\hline Presence of comorbidities* & & $19(76)$ & $24(66.7)$ & 0.43 \\
\hline \multirow[t]{2}{*}{ Histology } & High-grade serous & $100 \%$ & $97.1 \%$ & 0.39 \\
\hline & Endometrioid & & $2.9 \%$ & \\
\hline \multirow{13}{*}{$\begin{array}{l}\text { High grade } \\
\text { FIGO stage }\end{array}$} & & $25(100)$ & $34(94.4)$ & 0.48 \\
\hline & Stage 1 & 6 & 3 & 0.65 \\
\hline & IA & 2 & 0 & \\
\hline & IB & 2 & 1 & \\
\hline & IC & 2 & 2 & \\
\hline & Stage 2 & 1 & 4 & \\
\hline & IIA & 1 & 1 & \\
\hline & IIB & 0 & 3 & \\
\hline & Stage 3 & $15(58)$ & $21(55)$ & \\
\hline & IIIA & 0 & 2 & \\
\hline & IIIB & 1 & 2 & \\
\hline & IIIC & 14 & 17 & \\
\hline & Stage 4 & 4 & 10 & \\
\hline High CA125 & & $20(80)$ & $26(72.2)$ & 0.46 \\
\hline \multirow[t]{2}{*}{ Initial management } & PDS & $48 \%$ & $50 \%$ & 0.87 \\
\hline & NAC & $52 \%$ & $50 \%$ & \\
\hline No. of lines, median (IQR) & & $3(1-5)$ & $3(2-4.75)$ & 0.79 \\
\hline \multicolumn{5}{|l|}{ Lines of treatment } \\
\hline First line $(n=61)$ & & $(n=25)$ & $(\mathrm{n}=18)$ & \\
\hline PBC & & $100 \%$ & $100 \%$ & 0.26 \\
\hline Non PBC & & & & \\
\hline Second line $(n=49)$ & & $(n=18)$ & $(\mathrm{n}=31)$ & \\
\hline PBC & & 88.9 & 83.4 & 0.79 \\
\hline Non PBC & & 11.1 & 16.6 & \\
\hline Third line $(n=39)$ & & $(n=13)$ & $(n=26)$ & \\
\hline $\mathrm{PBC}$ & & 73.1 & 64 & 0.42 \\
\hline Non PBC & & 26.9 & 36 & \\
\hline Fourth line $(n=23)$ & & $(n=10)$ & $(n=13)$ & \\
\hline PBC & & $50 \%$ & $23.1 \%$ & 0.38 \\
\hline Non PBC & & $50 \%$ & $76.9 \%$ & \\
\hline Fifth line $(n=13)$ & & $(n=7)$ & $(n=5)$ & \\
\hline PBC & & - & - & 0.68 \\
\hline Non PBC & & $100 \%$ & $100 \%$ & \\
\hline
\end{tabular}

Categorical values were compared with the Chi-square test or Fisher's exact test, and continuous values were described as the median with interquartile range (IQR) and compared using the Mann-Whitney U test

Comorbidities: hypertension, DM, hypothyroidism, bronchial asthma, dyslipidemia, or osteoarthritis. PBC; platinum-based chemotherapy; non PBC, non-platinum-based chemotherapy (Paclitaxel, Liposomal doxorubicin, Etoposide, Gemcitabine, 
Table 2. Patients, age and regions, family history, and deleterious mutations $(n=25)$

\begin{tabular}{|c|c|c|c|c|c|c|}
\hline Age & Region* & $\begin{array}{l}\text { Personal History of } \\
\text { cancer }\end{array}$ & $\begin{array}{l}\text { Family members/ type of } \\
\text { cancer }\end{array}$ & Gene & Mutation & Protein change \\
\hline 60 & South & - & - & BRCA1 & c1140dupG & p.Lys381Glufs*3 \\
\hline 38 & Central & - & - & BRCA1 & c.2410_2413del & p.gln804Valfs*10 \\
\hline 56 & Southern & - & Sister (breast) & BRCA1 & c.1426_1433del & p.His476* \\
\hline 45 & Central & Breast Ca & - & BRCA1 & c.1140dupG & p.Lys381Glufs*3 \\
\hline 49 & Central & Breast Ca & - & BRCA1 & c.5530del & p.Leu1844Serfs*11 \\
\hline 52 & Western & Breast Ca & Sister (breast) & BRCA1 & c.1140dupG & p.Lys381Glufs*3 \\
\hline 52 & Central & - & - & BRCA1 & c.1140dupG & p.Lys381Glufs*3 \\
\hline 41 & Central & - & - & BRCA2 & c.5762_5772del & p.Phe1921Serfs*3 \\
\hline 62 & Northern & - & - & BRCA1 & c. $5074+2 \mathrm{~T}>\mathrm{T}$ & \\
\hline 50 & Northern & - & - & BRCA1 & c. $5095 \mathrm{C}>\mathrm{T}$ & p.Arg1699Trp \\
\hline 48 & Western & - & - & BRCA1 & c.1140dupG & p.lys 381Glufs*3 \\
\hline 40 & Eastern & - & - & BRCA1 & c1140dupG & p.Lys381Glufs*3 \\
\hline 53 & Central & - & $\begin{array}{l}\text { Sister (breast/ovarian) } \\
\text { Father (Colon) }\end{array}$ & BRCA1 & c.135-1 del & \\
\hline 45 & Central & - & $\begin{array}{l}\text { Sister (breast/ovarian) } \\
\text { Father (Lung) }\end{array}$ & BRCA1 & c.1140dupG & p.Lys 381 Glufs*3 \\
\hline 43 & Central & Breast Ca & Mother and Sister (breast) & BRCA1 & c.1140dupG & p.Lys381Glufs*3 \\
\hline 48 & Central & $\begin{array}{l}\text { Pheochromocytoma, } \\
\text { Breast Ca }\end{array}$ & Brother (colon Ca) & BRCA1 & c.2405_2406del & p.Val802Glufs*7 \\
\hline 43 & Central & - & - & BRCA1 & c.1016del & p.Lys339Argfs*2 \\
\hline 56 & Central & - & - & BRCA1 & c.69del & p.Glu23Valfs*17 \\
\hline 56 & Central & - & Sister (breast) & BRCA1 & c. $5095 \mathrm{C}>\mathrm{T}$ & p.Arg1699Trp \\
\hline 69 & Southern & - & - & BRCA1 & c. $2572 \mathrm{C}>\mathrm{T}$ & p.Gln858* \\
\hline 41 & Eastern & - & - & BRCA1 & c.1140dupG & p.Lys381Glufs*3 \\
\hline 67 & Central & - & - & BRCA1 & c.5530del & p.Leu1844Sarfs*11 \\
\hline 35 & Southern & - & - & BRCA1 & c.708_711dupTGAA & p.His228* \\
\hline 59 & Northern & - & - & BRCA1 & c.5530del & p.Leu1844Serfs*11 \\
\hline 50 & Northern & - & - & BRCA2 & c. $7007 \mathrm{G}>\mathrm{A}$ & p.Arg2336His \\
\hline
\end{tabular}

Regions according to the Saudi cancer registry: Central region (Riyadh, Qassim, and Hail), Northern region (Madinah, Tabuk, Jouf, and Northern), Western region (Makkah, Madinah, Jeddah, and Taif), Eastern region (Dammam and Ahsa), and Southern region (Jizan, Naran, Baha, and Asir

Table 3. Response rate to different lines of chemotherapy based on BRCA status. 


\begin{tabular}{|c|c|c|c|c|c|c|c|c|}
\hline \multirow[t]{2}{*}{ Best Response } & \multicolumn{2}{|c|}{ First line $(n=61)$} & \multicolumn{2}{|c|}{ Second line $(n=49)$} & \multicolumn{2}{|c|}{ Third line $(\mathrm{n}=39)$} & \multicolumn{2}{|c|}{ Fourth line $(n=23)$} \\
\hline & $\begin{array}{c}\text { BRCAm } \\
\mathrm{n}=25\end{array}$ & $\begin{array}{l}\text { BRCAw } \\
\mathrm{n}=36\end{array}$ & $\begin{array}{c}\text { BRCAm } \\
\mathrm{n}=18\end{array}$ & $\begin{array}{c}\text { BRCAw } \\
\mathrm{n}=31\end{array}$ & $\begin{array}{c}\text { BRCAm } \\
\mathrm{n}=13\end{array}$ & $\begin{array}{l}\text { BRCAw } \\
n=26\end{array}$ & $\begin{array}{c}\text { BRCAm } \\
\mathrm{n}=10\end{array}$ & $\begin{array}{l}\text { BRCAw } \\
n=13\end{array}$ \\
\hline CR & $92 \%$ & $72.7 \%$ & $50 \%$ & $29 \%$ & $46.2 \%$ & $7.7 \%$ & $20 \%$ & $7.7 \%$ \\
\hline PR & $8 \%$ & $27.3 \%$ & $44.4 \%$ & $35.5 \%$ & $38.5 \%$ & $19.2 \%$ & $20 \%$ & $7.7 \%$ \\
\hline SD & - & - & $5.6 \%$ & $9.7 \%$ & - & $26.9 \%$ & $10 \%$ & $15.4 \%$ \\
\hline PD & - & - & - & 25.8 & $15.4 \%$ & $34.6 \%$ & $50 \%$ & $38.5 \%$ \\
\hline NA & - & 3 patients & - & - & - & 3 patients & - & 4 patients \\
\hline ORR & $100 \%$ & $100 \%$ & $94.4 \%$ & $64.5 \%$ & $84.7 \%$ & $30.6 \%$ & $40 \%$ & $22.2 \%$ \\
\hline P-Value* & & & & & & 002 & & .40 \\
\hline
\end{tabular}

* Chi-square or Fisher's exact test ; CR, complete response; PR, partial response; SD, stable disease; PD, progressive disease; NA, not available; ORR, Objective response rate

\section{Figures}




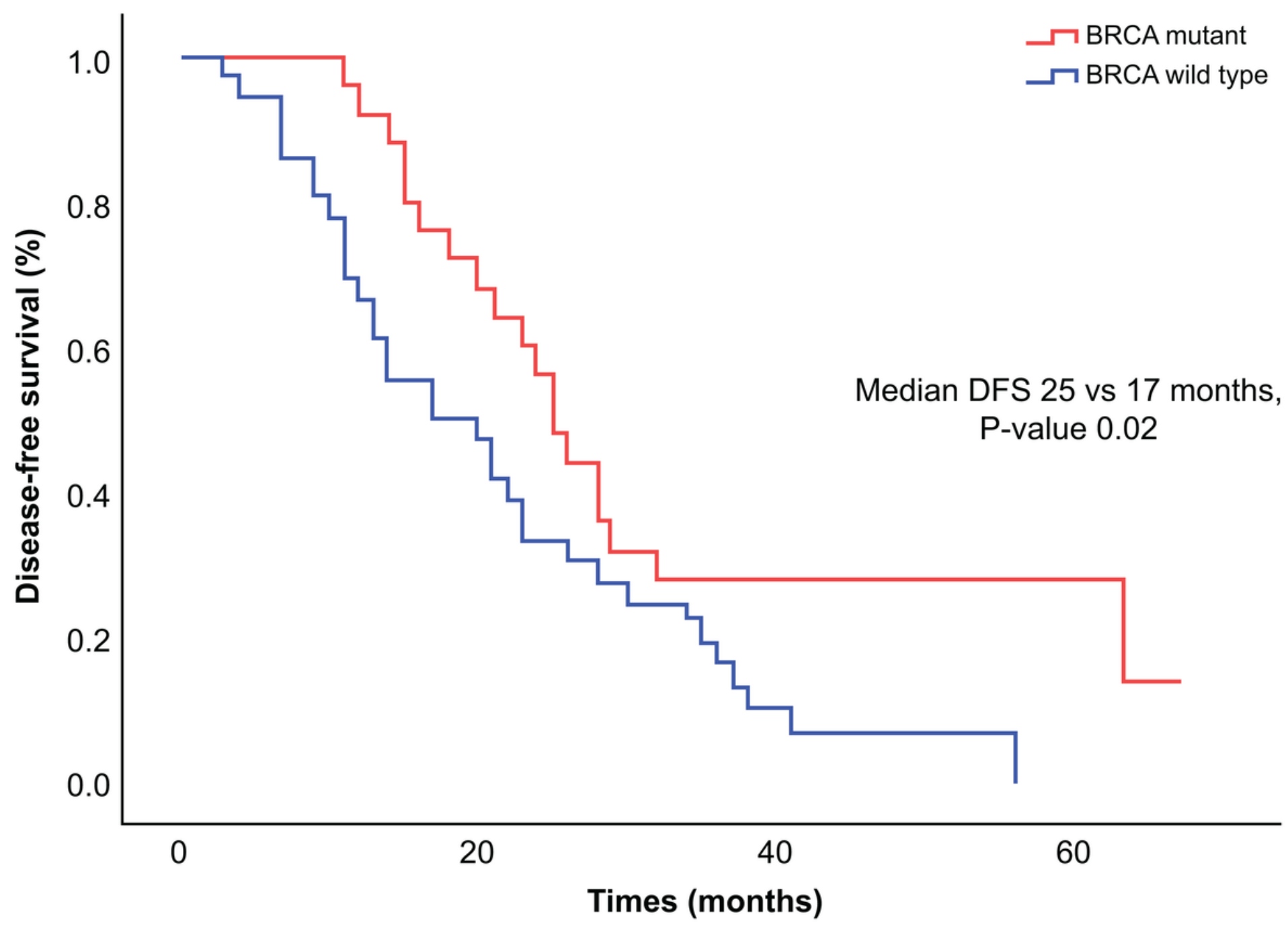

\section{Figure 1}

Disease-free survival of ovarian cancer stratified by BRCA status for first-line therapy. The Kaplan-Meier estimator was used to determine DFS among patients with germline BRCA mutation and those without germline BRCA mutation.Two sided $\mathrm{P}$ values were calculated with the use of the stratified log rank test and $\mathrm{Cl}$ denotes confidence interval. The median DFS was longer in the BRCA mutant women 25 (95\% $\mathrm{Cl}$ : $21.7-28.2)$ vs. $17(95 \% \mathrm{Cl}: 8.7-25)$ months, $(p=0.02)$. 


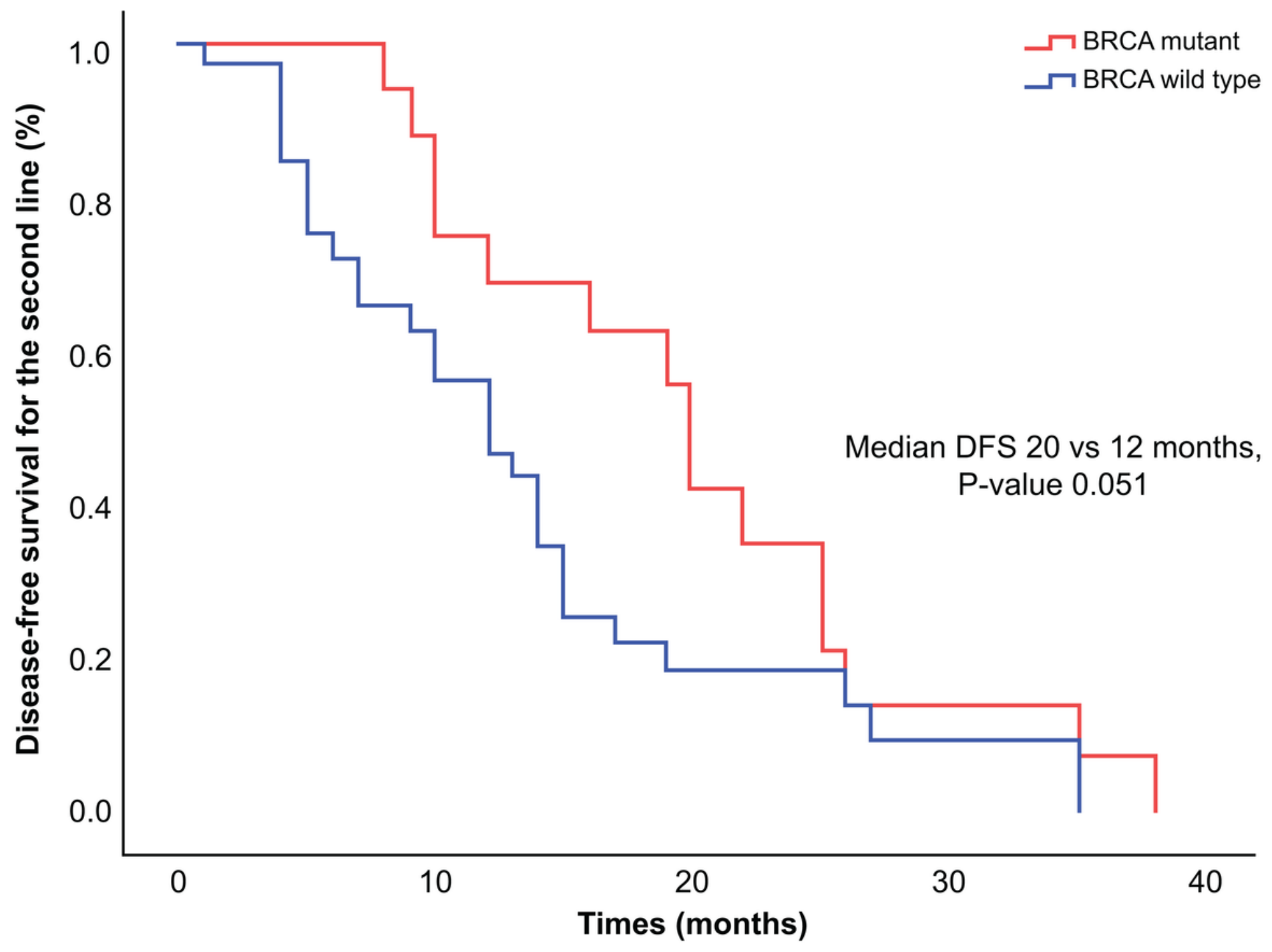

Figure 2

Disease-free survival of ovarian cancer stratified by BRCA status for second-line therapy. The KaplanMeier estimator was used to determine DFS for second line therapy among patients with germline BRCA mutation and those without germline BRCA. Two sided $P$ values were calculated with the use of the stratified log rank test and $\mathrm{Cl}$ denotes confidence interval. The median DFS of second-line treatment (50 patients) in the BRCA mutant group was 20 months (95\% Cl: 18.2-21.7) vs. 12 months (95\% Cl: 7.8$16.1)$ in the wild-type group $(p=0.051)$ 


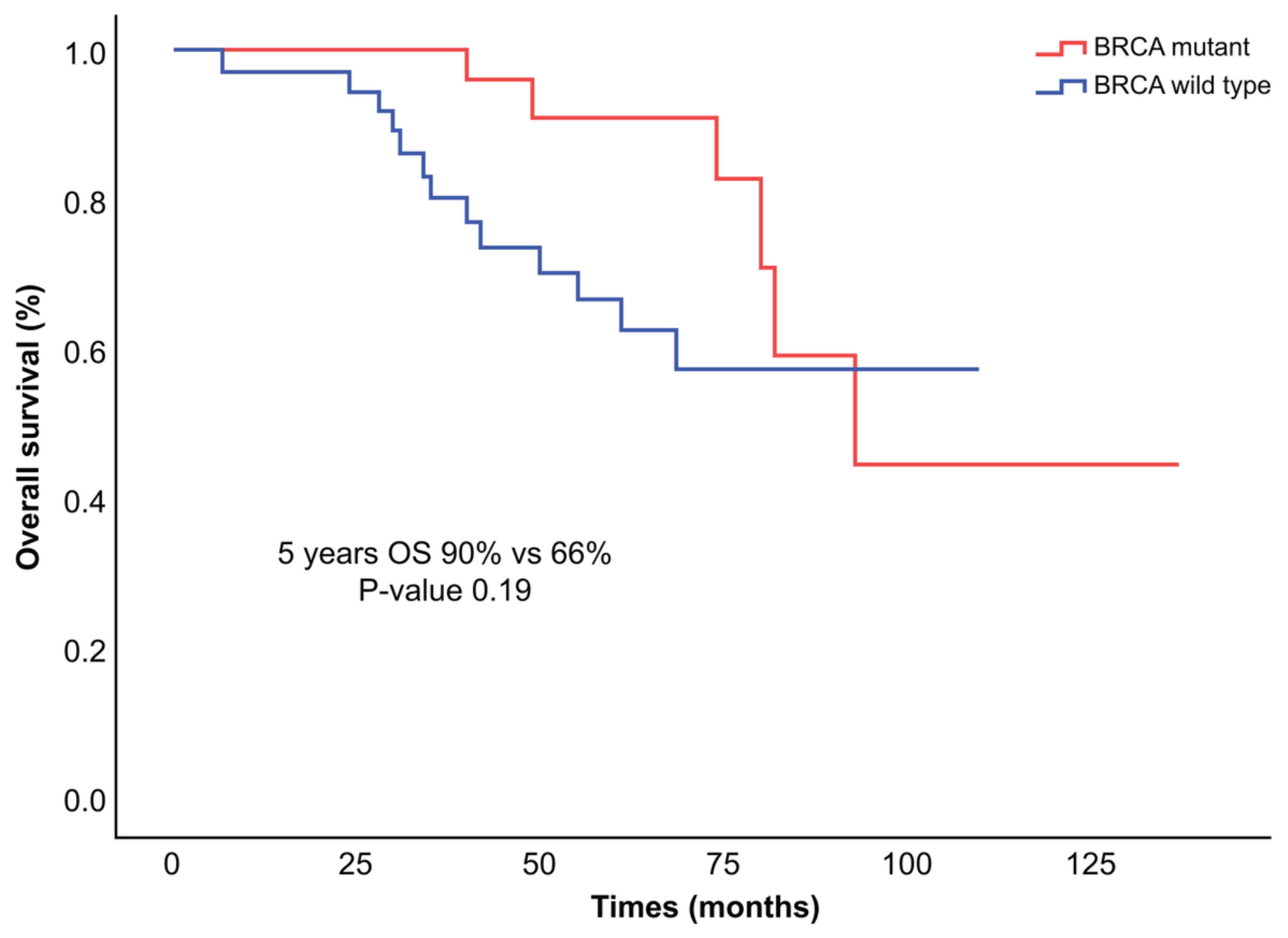

\section{Figure 3}

Five-year overall survival of ovarian cancer stratified by BRCA status. The Kaplan-Meier estimator was used to determine OS among patients with germline BRCA mutation and those without germline BRCA mutation. Two sided $\mathrm{P}$ values were calculated with the use of the stratified log rank test and $\mathrm{Cl}$ denotes confidence interval. The median OS was not reached. However, the five-year OS rate for BRCA mutant patients was $90.9 \%$ vs. $66.7 \%$ for wild-type patients $(p=0.19)$

\section{Supplementary Files}

This is a list of supplementary files associated with this preprint. Click to download.

- FCSTROBEchecklistv4combined.docx 